\title{
Variedades Anatómicas del Árbol Biliar. Implicancia Quirúrgica
}

\author{
Anatomy Variations of Biliary Tree. Surgical Implicance
}

*;***Mariano José Tolino; ****Ana Sofía Tartaglione; **Carlos Diego Sturletti \& **Mariana Ivon García

TOLINO, M. J.; TARTAGLIONE, A. S.; STURLETTI, C. D. \& GARCÍA, M. I. Variedades anatómicas del árbol biliar. Implicancia quirúrgica. Int. J. Morphol., 28(4):1235-1240, 2010.

RESUMEN: La anatomía de las vías biliares presenta frecuentes variaciones. La anatomía "normal" se describe en aproximadamente la mitad de los casos. Las variedades de las vías biliares deben ser conocidas por los médicos especialistas en diagnóstico por imágenes y cirujanos hepatobiliares, a fin de optimizar su desempeño en sus respectivas áreas. Con ese fin fueron estudiadas en forma prospectiva 690 colangiografías (538 intra-operatorias; 90 resonancias; y 62 trans-catéteres biliares). Se utilizó la clasificación de Blumgart y los resultados fueron: tipo A: 284 (41,16\%); B: 178 (25,80\%). C1: 110 (15,94\%); C2: 38 (5,51\%); D1: 8 (1,16\%); D2: 23 (3,33\%); E1: 18 $(2,61 \%)$; E2: 27 (3,91\%); F: 4 (0,58\%). Como conclusiones, es importante señalar que: a) la colangiografía intra-operatoria debe ser sistemática, por un lado, para descartar litiasis coledociana; y por el otro, para detectar variaciones anatómicas y predecirlas antes de las cirugías resectivas; y b) la colangiorresonancia es ideal como estudio preoperatorio en posibles donantes vivos de órganos.

PALABRAS CLAVE: Vías biliares; Anatomía; Cirugía; Colangiografías.

\section{INTRODUCCIÓN}

La anatomía de los conductos biliares sigue la segmentación del sistema portal en el hígado. Sin embargo, apenas la mitad presentan la llamada "anatomía normal". Algunos señalan variantes entre 24-57\% (Castaing, 2008; D’Ippolito et al., 2006; Healey \& Schroy, 1953).

El conocimiento de las variantes anatómicas se ha revalorizado con el advenimiento de la cirugía laparoscópica de vesícula, las operaciones por tumores hepato-biliares y los transplantes hepáticos.

La colangiografía intra-operatoria en cirugía laparoscópica, es fundamental para detectar variaciones ductales y su relación con las maniobras quirúrgicas a fin de prevenir o detectar precozmente lesiones quirúrgicas (Chiappeta Porras et al., 2000).

La colangiorresonancia magnética nuclear, es útil en el preoperatorio de la cirugía de transplante hepático (D’Ippolito et al.) y es el método no invasivo de elección para visualizar las vías biliares.

* Hospital Zonal de Trelew "Adolfo Margara", Trelew, Chubut ,Argentina.

* Sanatorio Franchin, Ciudad Aut. de Buenos Aires, Argentina.

**** Instituto de Diagnóstico del Este de Chubut, Trelew, Chubut, Argentina.
El objetivo de este trabajo es determinar la frecuencia de las principales variantes anatómicas y discutir su relación con la cirugía.

\section{MATERIAL Y MÉTODO}

En tres instituciones: Sanatorio Franchín, de Buenos Aires; Hospital Zonal de Trelew, Chubut; y el Instituto de Diagnóstico del Este de Chubut, Argentina, entre Agosto2005 y Octubre-2009 se recogieron en forma prospectiva 706 imágenes de colangiografías obtenidas con tres métodos diferentes:

a- 548 colangiografías intraoperatorias (CIO), que se realizaron introduciendo un catéter flexible de polivinilo e inyectando lentamente de 20 a $40 \mathrm{ml}$ de sustancia de contraste iodado diluido al $30 \%$, visualizando la repleción biliar por radioscopía mediante intensificador de imágenes portátil arco en $\mathrm{C}$. 
b- 96 colangiorresonancias (CR), realizadas con un equipo Signa 1.5 T (General Electric);

c- 62 colangiografías trans-catéteres biliares (CTC), también controlados por radioscopía mediante inyección de sustancia de contraste iodado.

La imágenes fueron interpretadas por un médico especialista en diagnóstico por imágenes y un cirujano hepatobiliar. Se agruparon de acuerdo a la clasificación de variedades de las vías biliares de Blumgart (Hahn \& Blumgart, 2000) (Fig. 1).
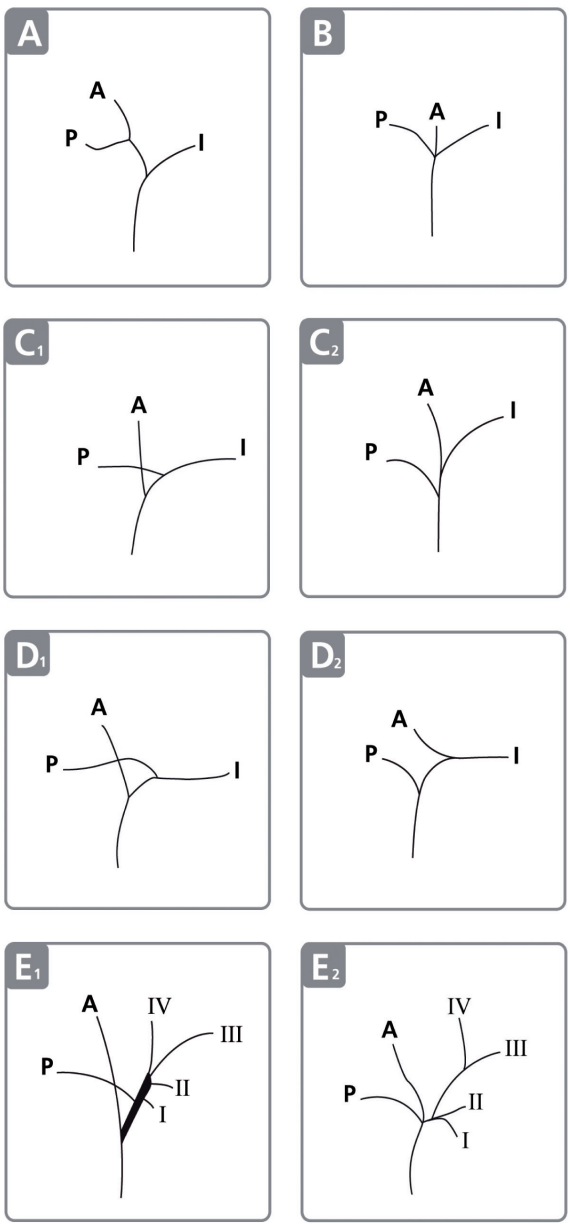

Fig. 1. Clasificación de Blumgart de las variaciones biliares.

\section{RESULTADOS}

Siguiendo la clasificación de Blumgart, se los agrupó en nueve tipos. Fueron descartados 20 estudios (10 CIO y $6 \mathrm{CR}$ ) porque las imágenes no permitieron observar adecuadamente la confluencia de los conductos. El total analizado fue de 690 colangiografías.

Tipo A: o "típica” anatomía ductal, corresponde a una confluencia izquierda-derecha y división de ramos derechos anterior y posterior (Fig. 2).

Tipo B: Triple confluencia de ramos izquierdo, derecho anterior y derecho posterior (Fig. 3).

Tipo C1: Desembocadura del conducto hepático derecho anterior en el conducto hepático común (Fig. 4).

Tipo C2: Desembocadura del conducto hepático derecho posterior en el conducto hepático común (Fig. 5).

Tipo D1: Desembocadura del conducto hepático derecho posterior en el conducto hepático izquierdo (Fig. 6).

Tipo D2: Desembocadura del conducto hepático derecho anterior en el conducto hepático izquierdo (Fig. 7).

Tipos E1 y E2: Ausencia de confluencia de los conductos hepáticos (Fig. 8).

Tipo F: Drenaje del conducto hepático derecho posterior a la altura o dentro del conducto cístico (Fig. 9).

La Tabla I resume los hallazgos de nuestro estudio en cantidad de casos y porcentuales de las distintas variaciones.

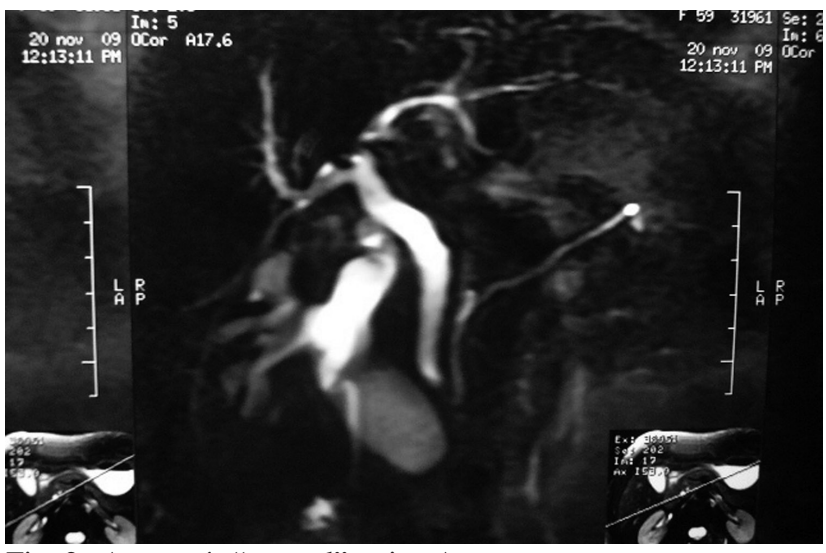

Fig. 2. Anatomía "normal" o tipo A. 


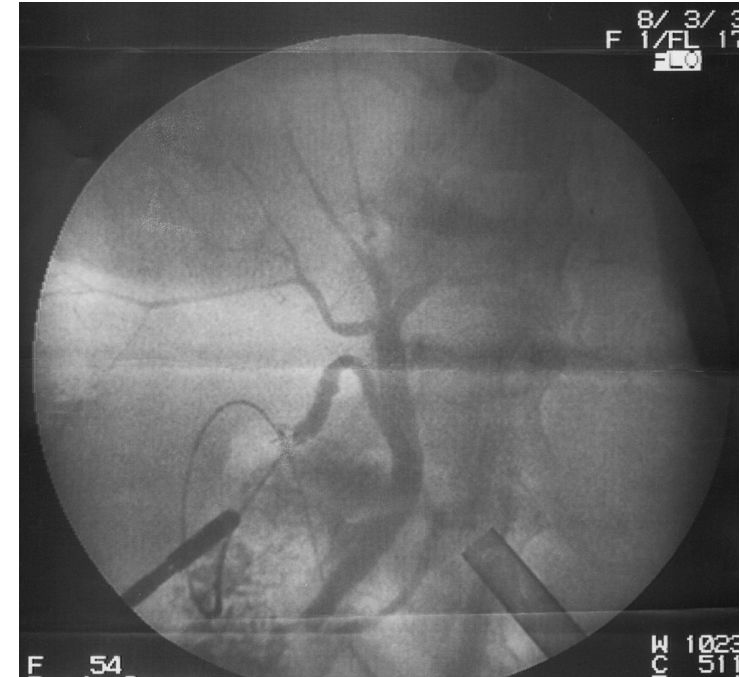

Fig. 3. Variedad tipo B, triple confluencia.

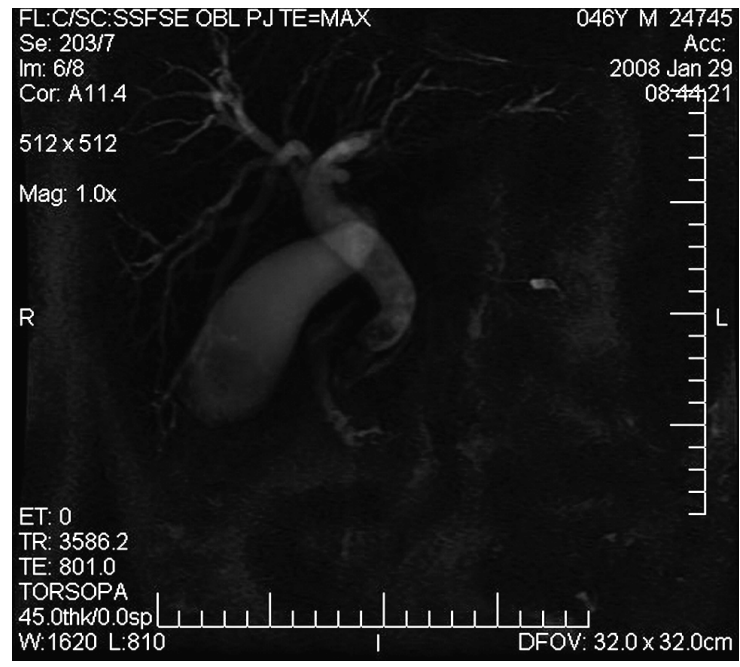

Fig. 4. Variedad tipo C1.

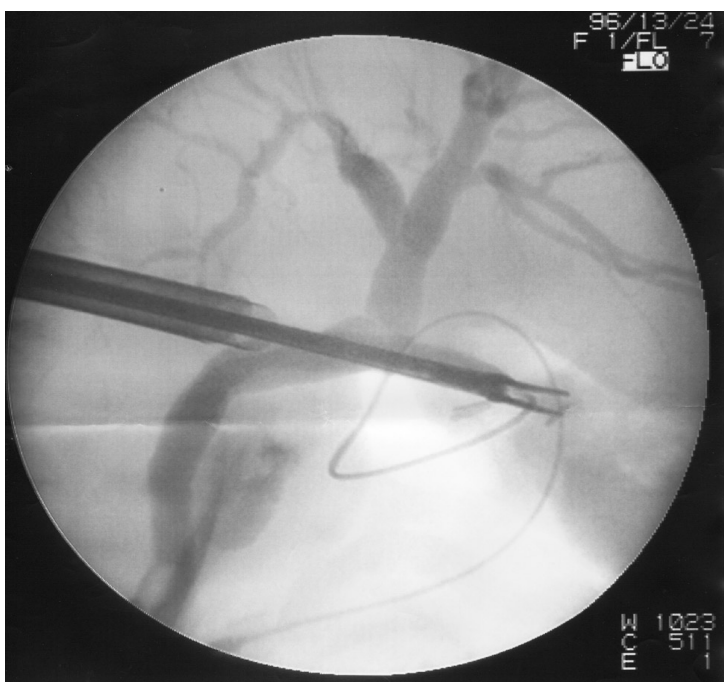

Fig. 5. Variedad tipo C2.

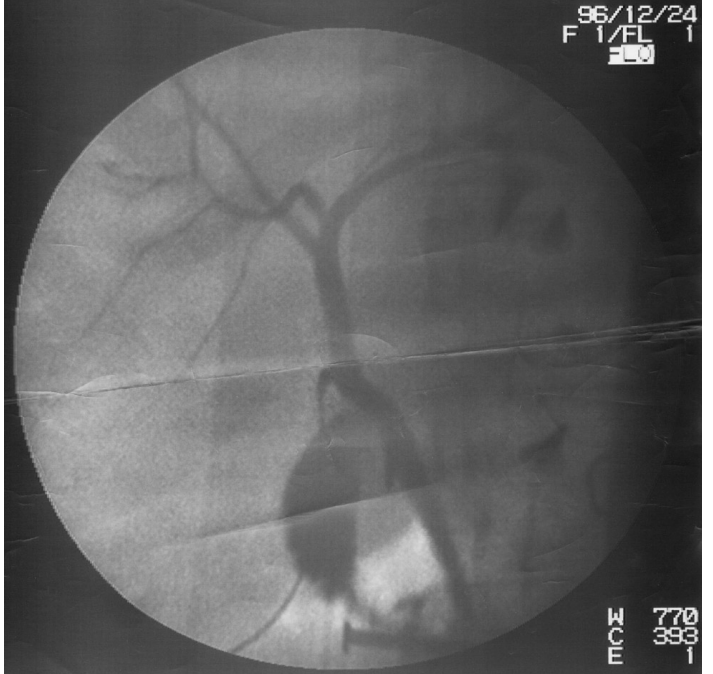

Fig. 6. Variedad tipo D1.

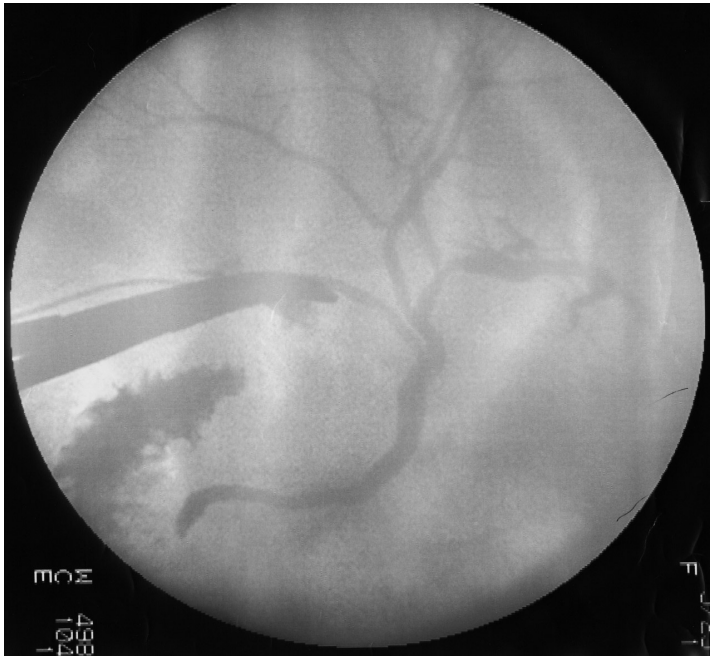

Fig. 7. Variedad tipo D2.

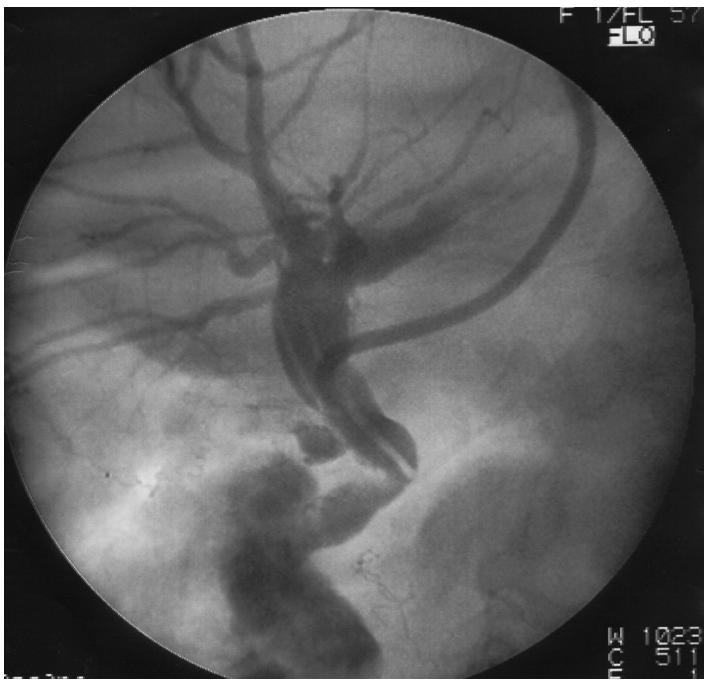

Fig. 8. Variedad tipo E. Ausencia de confluencia. 
Tabla I. Frecuencia de las variaciones biliares halladas en nuestra serie.

$\begin{array}{cccccccccc} & \text { A } & \text { B } & \text { C 1 } & \text { C 2 } & \text { D 1 } & \text { D 2 } & \text { E 1 } & \text { E 2 } & \text { F } \\ \text { Casos } & 284 & 178 & 110 & 38 & 8 & 23 & 18 & 27 & 4 \\ \text { \% } & 41,16 & 25,80 & 15,94 & 5,51 & 1,16 & 3,33 & 2,61 & 3,91 & 0,58\end{array}$

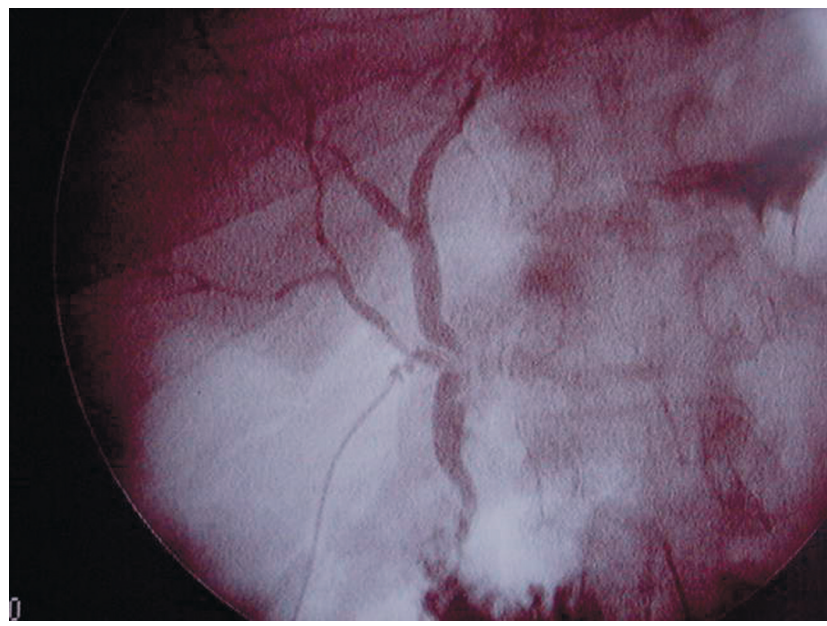

Fig. 9. Variedad tipo F. Drenaje bajo del derecho posterior al conducto cístico.

\section{DISCUSIÓN}

Anatomía biliar. La "anatomía normal", Tipo A de Blumgart, se presenta en aproximadamente la mitad de los casos. Nari et al., en un estudio basado en CIO, lo encontró en el $59 \%$. Castaign, en una revisión, lo describe en hasta el $82 \%$. En nuestra serie, fue encontrada en el $41 \%$. Las variedades anatómicas de la confluencia biliar son frecuentes; se las ha descripto entre 19-57\% (Champentier et al., 1989; Nari et al., 2005; Kullman et al., 1996; D’Ippolito et al.).

Colangiografía intraoperatoria. La CIO, ideada y descripta por Mirizzi (1939) se utiliza en colecistectomía laparoscópica para detectar litiasis coledociana y anomalías biliares. Si bien algunos autores apoyan su realización selectiva (Metcalfe et al., 2004; Bregante et al., 2006; Lorimer \& Fairfull-Smith, 1995), creemos, junto a otros, que ésta debe realizarse sistemáticamente, no sólo para descartar litiasis, sino principalmente para observar los ramos ductales y sus recorridos, a fin de prevenir la lesión accidental en la cirugía (Berci, 1992; Strasberg, 2005; Chiappeta Porras et al.; Kullman et al.).

En cuanto a la técnica para realizar la CIO, Nari et al. notaron que debieron excluir un gran porcentaje de $\mathrm{CIO}$ (159 de 497, 32\%), que atribuyeron a errores técnicos por:
1) introducción excesiva de la cánula; y 2) inyección rápida del contraste. Ambas maniobras facilitarían la evacuación rápida del contraste al duodeno y provocarían un insuficiente llenado de las vías biliares, sin aportar los datos necesarios. En nuestra serie, de 548 CIO se descartaron 10 $(1,82 \%)$ y ninguna de las colangiografías trans-catéteres.

Variedades Anatómicas - Implicancias quirúrgicas. Las anomalías de implantación de los conductos biliares derechos constituyen las variaciones más frecuentes de la VB y uno de los principales factores de riesgo de lesión quirúrgica ductal durante la colecistectomía (Alvarez Rodriguez et al., 2006).

Una variedad que puede generar complicaciones intra o postoperatorias es la desembocadura baja de un conducto hepático derecho posterior (C2 o D2), donde puede existir relación de éste con la fosa vesicular y ser lesionado durante la colecistectomía. Alvarez Rodriguez et al. describen 4 casos en 4850 colecistectomías $(0,08 \%)$ que debieron ser reoperados para su resolución. Maquillan et al. (1989) describieron 6 casos de conductos hepáticos accesorios en cadáveres y 4 casos clínicos resueltos con drenajes. En nuestra serie tuvimos 1 caso de un conducto hepático accesorio del segmento VI detectado y clipado durante la operación, sin complicaciones inmediatas ni en el seguimiento a 28 meses.

La variante $\mathrm{F}$, poco frecuente, es tal vez la de mayor riesgo de lesión durante una operación de vesícula, por su desembocadura dentro o cercana al conducto cístico. D'Ippolito et al. lo encontraron en un $1 \%$; Nari et al. en el $1,18 \%$. Anderson (1996), en un estudio en 95 cadáveres, encontró en 2 casos la desembocadura directa al conducto cístico $(2,10 \%)$. En nuestra serie, esta variante fue hallada en 4 casos, el $0,58 \%$ del total.

Transplantes hepáticos. La escasez de hígados cadavéricos para transplantes, y el aumento de posibles receptores, ha motivado el desarrollo de los transplantes con donante vivo. Para la selección de éstos, no sólo se tienen en cuenta factores inmunológicos de compatibilidad, sino también aquellos relacionados con su anatomía hepática. En transplantes, las variaciones biliares están asociadas a un aumento del riesgo de complicaciones, fístulas y estricturas (Lee et al., 2004; Cheng et al., 2007; Soares et al., 2006). 
Imágenes Por Resonancia: El estudio preoperatorio para seleccionar al donante vivo debe incluir un mapeo de las vías biliares; para ello, la colangiorresonancia (CR) es el método no invasivo ideal para su estudio. Sin embargo, el método no es infalible. Soares et al., en una serie de 82 pacientes, la mitad donantes y la otra receptores, realizaron 33 CR, detectando 3 variantes: una Tipo B y dos Tipo D1. Comparando estos resultados con las CIO durante las ablaciones e implantes, la CR tuvo una sensibilidad del $67 \%$ y especificidad del $100 \%$ para detectar variantes anatómicas. En un estudio similar, en 30 donantes vivos, Kim et al. (2005) obtuvieron una sensibilidad del $92 \%$ y especificidad del $100 \%$.

Con el fin de optimizar la resolución de las vías biliares por CR, se ha desarrollado una técnica con Mangafodipir trisódico, un contraste que se excreta por VB en fase temprana y es usado en T1-pesado ya que el manganeso se excreta como metal ion. Lee et al. compararon este nuevo método con el habitual en T2 sin contraste. La CR con Mangafodipir tuvo una sensibilidad del $60 \%$ y especificidad del $100 \%$ para detectar variantes; la CR sin contraste, $50 \%$ y $93 \%$ respectivamente.

En conclusión, las variedades anatómicas de las vías biliares son frecuentes y deben ser conocidas por los médicos que realizan diagnóstico por imágenes y cirujanos hepatobiliares, a fin de lograr un mejor diagnóstico y disminuir la morbilidad asociada a las cirugías del hígado y las vías biliares. Es importante resaltar que la CIO debe realizarse con mínima introducción de la cánula en el conducto y se debe inyectar lentamente el contraste a fin de visualizar todas las vías biliares.

TOLINO, M. J.; TARTAGLIONE, A. S.; STURLETTI, C. D. \& GARCÍA, M. I. Anatomy variations of biliary tree. Surgical implicance. Int. J. Morphol., 28(4):1235-1240, 2010.

SUMMARY: The anatomy of the biliary tract have frequent variations. Normal anatomy is described in about half of cases. The varieties of the bile ducts should be known by physicians and hepatobiliary surgeons specializing in diagnostic imaging, in order to optimize performance in their respective areas. To this end 690 cholangiographies (538 intra-operative, 90 resonances, and 62 transcatheter bile) were prospectively studied. We used the classification of Blumgart and the results were as follows: type A: 284 (41.16\%), B: 178 (25.80\%). C1: 110 (15.94\%), C2: 38 (5.51\%)., D1: 8 (1.16\%), D2: 23 (3.33\%), E1: 18 (2.61\%), E2: 27 (3.91\%), F: 4 (0.58\%). As conclusion, it is important to note that: a) intra-operative cholangiography should be systematic, on the one hand, to exclude cholelithiasis and, on the other hand, to detect and predict anatomical changes before resective surgery and $b$ ) cholangioresonance is an ideal preoperative study in potential living donors of organs.

KEY WORDS: Biliary tract; Anatomy; Surgery; Cholangiography.

\section{REFERENCIAS BIBLIOGRÁFICAS}

Alvarez Rodríguez, J.; Lorenzo, A.; Kohan, G.; Quesada, B. M.; Chiappetta Porras, L.; Canullán, C. \& Oría, A. Estrategia terapéutica en el diagnóstico postoperatorio de lesión de un conducto hepático anómalo. Rev. Argent. Cirug., 90:121-31, 2006.

Anderson, J. Grant Anatomía. Montevideo, Intermédica, 1986.

Berci, G. Biliary ductal anatomy and anomalies. The role of intraoperative cholangiography during laparoscopic cholecystectomy. Surg. Clin. North Am., 72:1069-75, 1992.

Bregante, M.; Pirchi, D.; Castagneto, G. \& Iribarren, C. La colangiografía intraoperatoria selectiva en colecistitis aguda. Rev. Argent. Cirug., 91:100-4, 2006.

Castaing, D. Surgical anatomy of the biliary tract. $H P B$ (Oxford), 10:72-6, 2008.
Champetier, J.; Letoublon, C.; Arvieux, C.; Gerard, P. \& Labrosse, P. A. Variations of division of the extrahepatic bile ducts: significance and origin, surgical implications. J. Chir., 126:147-54, 1989.

Cheng, Y. F.; Huang, T. L.; Chen, C. L.; Chen, Y. S. \& Lee, T. Y. Variations of the intrahepatic bile ducts: application in living related liver transplantation and splitting liver transplantation. Clin. Transplant., 11:337-40, 1997.

Chiappetta Porras, L.; Hernández, N.; Nápoli, E.; Romano, M.; Canullán, C., Fernández, G. \& Oría, A. Importancia de las variantes anatómicas de la vía biliar en la era laparoscópica. Rev. Argent. Cirug., 78:87-95, 2000.

D’Ippolito, G.; de Queiroz Rosas, G.; Appezzato, L. F.; Araújo Carvalho, G. \& de Melo Galvao Filho, M. Variantes anatómicas e anomalías congénitas das vías biliares e pancreáticas: análise através de colangiopancreatografía por ressonancia magnética. Rev. Imagen, 28:33-9, 2006. 
TOLINO, M. J.; TARTAGLIONE, A. S.; STURLETTI, C. D. \& GARCÍA, M. I. Variedades anatómicas del árbol biliar. Implicancia quirúrgica. Int. J. Morphol., 28(4):1235-1240, 2010.

Hahn, L. \& Blumgart, L. H. Surgical and radiologic anatomy of the liver and biliarytree. In: Surgery of the Liver and Biliary Tract. Blumgart, L. H. \& Fong, Y. (Eds.). London, W.B. Saunders, 2000.

Healey, J. E. Jr. \& Schroy, P. C. Anatomy of the biliary ducts within the human liver; analysis of the prevailing pattern of branchings and the major variations of the biliary ducts. AMA Arch. Surg., 66:599-616, 1953.

Kim, R. D.; Sakamoto, S.; Haider, M. A.; Molinari, M.; Gallinger, S.; McGilvray, I. D.; et al. Role of magnetic resonance cholangiography in assessing biliary anatomy in right lobe living donors. Transplantation, 79:141721, 2005.

Kullman, E.; Borch, K.; Lindström, E., Svanvik, J. \& Anderberg, B. Value of routine intraoperative cholangiography in detecting aberrant bile ducts and bile duct injuries during laparoscopic cholecystectomy. $\mathrm{Br}$. J. Surg., 83:171-5, 1996.

Lee, V. S.; Krinsky, G. A.; Nazzaro, C. A.; Chang, J. S.; Babb, J. S.; Lin, J. C.; et al. Defining intrahepatic biliary anatomy in living liver transplant donor candidates at mangafodipir trisodium-enhanced MR cholangiography versus conventional T2-weighted MR cholangiography. Radiology, 233:659-66, 2004.

Lorimer, J. W. \& Fairfull-Smith, R. J. Intraoperative cholangiography is not essential to avoid duct injuries during laparoscopic cholecystectomy. Am. J. Surg., 169:344-7, 1995.

Metcalfe, M. S.; Ong, T.; Bruening, M. H.; Iswariah, H.; Wemyss-Holden, S. A. \& Maddern, G. J. Is laparoscopic intraoperative cholangiogram a matter of routine? Am. J. Surg., 187:475-81, 2004.

Maquillan, T.; Manolas, S. G.; Hayman, J. A. \& Kune, G. A. Surgical significance of the bile duct of Luschka. Br. J. Surg., 76:696-8, 1989.

Mirizzi, P. Fisiopatología del hepatocolédoco, Colangiografía intraoperatoria. Buenos Aires, El Ateneo, 1939.

Nari, G. A.; Jozami, M. L. \& Ponce, O. H. Variantes anatómicas del carrefour biliar. Análisis de 338 mirizzigrafías. Rev. Argent. Cirug., 89:19-22, 2005.

Soares, R. V.; Coelho, J. C.; Matias, J. E.; Freitas, A. C.; Zeni-Neto, C. \& Godoy, J. L. Anatomía das vías biliares em doadores e receptores de transplante hepático inter vivos. Arq. Gastroenterol., 43:173-7, 2006.

Strasberg, S. Biliary injury in laparoscopy surgery. Part 1-2. Am. Col. Surg., 4:458-603, 2005.

Dirección para correspondencia:

Mariano J. Tolino

A. P. Bell $520(9100)$

Trelew

Chubut

ARGENTINA

Email: marianotolino@yahoo.com.ar

Recibido : 29-07-2010

Aceptado: 15-11-2010 